\title{
FINANCIAL MODELS WITH DEPENDENCE ON THE PAST: A SURVEY
}

\author{
V. HALLULLI AND T. VARGIOLU* \\ Department of Pure and Applied Mathematics \\ University of Padova \\ via Belzoni 7, I-35131 Padova - Italy \\ phone: +390498275883 \\ fax: +390498275892 \\ E-mail: hallulli@math.unipd.it, \\ vargiolu@math.unipd.it
}

\begin{abstract}
In this paper we will examine some models for the financial markets where the evolution of the prices of the assets depends not only on the current value but also from the values assumed in the past. First we characterise the shape of volatility that a "good" financial model, also depending on past values, must have, and prove the completeness of the market in this general framework. Then, we analyse two models taken from the literature, one with "finite memory" which in general does not give nice numerical results, and one with "infinite memory", where the model reduces to a Markov system with more state variables than the number of risky assets. Finally, we prove that a generalisation of the results of this last model with finite delay horison is not feasible without using anticipative stochastic calculus.
\end{abstract}

\section{Introduction}

The year 1973 is a milestone in the modeling of financial markets: in fact, in that year the papers of Black and Scholes ${ }^{1}$ and $\mathrm{Merton}^{2}$, where an explicit formula for the price of call and put options was present, saw the light. The formula now known universally as "the Black and Scholes formula" links the price of a call option to quantities which are observed in the market (current price, strike price, time to maturity) and a parameter, the volatility, which gives an idea of how rapidly the asset prices can change. Moreover, they prove that it is possible to replicate exactly the final payoff of the call option by a dynamic hedging on the underlying asset and another "risk free" asset. The two papers cited above influenced financial markets

*corresponding author. 
so deeply that every investment bank today has to deal with "the Black and Scholes approach": this is also witnessed by the Nobel prize in 1997.

The so-called "Black and Scholes model" is however valid only as a first approximation: in fact, it was soon realised that the assumption of a constant volatility was in contrast with the empirical observations of derivative prices in real markets, which suggest that the volatility is not constant, but rather depends both on time to maturity and on the strike price. For this reason, during the following years many attempts have been made in order to formulate models for asset prices which fit the market price of derivatives better than the original Black and Scholes model with constant volatility. All these models can be split into two broad families: models with stochastic volatility and models with level-dependent volatility (a brief survey can be found in Ref. 3). In the first class of models, the volatility is a stochastic process which is driven by the asset price and by another source of randomness, and the market is usually incomplete. Conversely, in the second class of models the volatility is usually a function of time and current price level, and the market is complete. Moreover, in principle one can specify the volatility function in such a way that the prices of all the calls in the market coincide with the theoretical price computed by the model ${ }^{4,5}$. The drawback is that, to achieve this, one should be able to observe the prices of all the call options with any given maturity and strike (that is, one should observe infinitely many assets in the market), where in real markets only a finite number of assets is observed.

In order to take advantage from the power of level-dependent volatility models (in particular from the completeness of the market), in the last years a growing interest has been raised for market models where the asset prices' dynamics do not depend only on their current values, but also on past values: these models can be usually seen as generalisations of leveldependent volatility models, as the volatility is still a function of (time and) the asset prices.

This paper wants to be an analysis of what a "good" financial model, also depending on the past, should be, and a survey of some models presented in recent years. We now describe its structure more in detail.

Our first result in Sec. 2 is the characterisation of the risky asset's dynamics in the case of a diffusion model: this result is essentially a characterisation of the volatility of the risky asset for a financial model to have some good properties. In Sec. 3, we present an explicit proof of the completeness of the market in a rather general framework, where the only assumption that is made is that the only source of uncertainty in the market is the 
Brownian motion which drives the risky asset. In order to see some practical examples, we turn our attention to some models present in literature and in particular we analyse the problem of pricing and hedging a simple European contingent claim.

In Sec. 4 we analyse a model where the volatility depends on a single value of the price, "delayed" of a fixed time $r^{6}$. Unfortunately this simple idea does not lead to simple calculations: in fact, if the time to maturity is greater than the delay $r$, this model implies multiple integrations which gives explicit results in general only by resort of Monte Carlo methods.

In Sec. 5 we take into account the Hobson-Rogers model, where a dependence of the volatility on the entire past price path is proposed ${ }^{3}$. It is possible to show that the model can be made Markovian by adding suitable state variables, thus the calculation of the price of derivative assets can be reduced to the solution of a linear parabolic PDE. In this model, however, some theoretical issues about the sensitivity of the model to the (necessarily wrong) initial conditions still remain to be treated.

In Sec. 6 we present a naive proposal to put together the intuitiveness of the first model with the nice results of the second one, by modifying, at the light of the characterisation of Sec. 2, a model in Ref. 7 in a financial setting. Unfortunately, the system is no more Markov in the currently observed variables, unlike in the Hobson-Rogers model. Following Ref. 7, we check if the pricing and hedging problem can be however reduced to the solution of a PDE, as in the Hobson-Rogers model, regardless of the non-Markovianity of the system. We prove that this is possible if and only if the volatility depends on the current price only (that is, if and only if we have a Black-Scholes type model).

\section{The general framework}

We consider for simplicity a financial market with a riskless asset and a single risky asset, where the riskless asset is supposed to be constantly equal to 1 (this can be achieved by considering discounted or forward prices ${ }^{8}$ ), and the risky asset follows a continuous time dynamics that can be described, by speaking the language of stochastic differentials, by

$$
d S(t)=\Sigma(t) d W(t), \quad t \in[0, T]
$$

where $W$ is a standard Brownian motion under a so-called equivalent martingale measure $\mathbb{Q}$, and $T$ is a given time horizon. From now on, we assume that $\Sigma$ is a generic stochastic process such that the filtration generated by $S$ coincides with the one generated by $W$ (let us call this common filtration 
$\left.\left(\mathcal{F}_{t}\right)_{t \in[0, T]}\right)$ : this is a mathematical formalism to mean that the information available by the knowledge of the prices $S$ or of the values of $W$ up to time $t$ coincide.

Now we start to impose some constraint on the process $\Sigma$ : the first two assumptions that we want to impose for the model to be realistic are

i) that the future does not influence the dynamics in the present;

this is mathematically translated into asking that

$\Sigma$ is adapted to $\left(\mathcal{F}_{t}\right)_{t}$;

ii) that the prices and the diffusion coefficient are positive, i.e. that $S(t)>0$ and $\Sigma(t)>0$ a.s. for all $t \in[0, T]$.

In order to have a parsimonious model, we suppose that $\Sigma$ only depends on $S$ (and not explicitly on $W$ ). Then Assumption (1) implies that $\Sigma(t)$ is of the form

$$
\Sigma(t)=S(t) \sigma\left(t, S_{t}\right)
$$

where for every $t \in[0, T], \sigma(t, \cdot)$ is a deterministic function of $S_{t}$, which is the path of $S$ up to time $t$ (notice the difference between the current price $S(t)$ and the whole sequence $S_{t}$ of prices before time $t$ ). More explicitly, for all $t \in[0, T], S_{t}$ is a continuous (random) function defined as

$$
S_{t}(x):=S(t+x) \quad \forall x \in(-R, 0)
$$

where $R$ is a certain observation window. We can have two cases: $R=+\infty$ (infinite horizon) or $R \in[0,+\infty)$ (finite horizon). Notice that $S_{t}(0)=S(t)$. Moreover, if $R=0$ (i.e. the observation window is limited to the current time only), then we have the usual Markovian case, since $S_{t}$ coincides with $S(t)$, and $\sigma$ is a deterministic function of the price $S(t)$. With this specification, the risky asset's dynamics is specified as

$$
d S(t)=S(t) \sigma\left(t, S_{t}\right) d W(t), \quad t \in[0, T],
$$

which is similar to the well-known Black-Scholes model, with the difference that the volatility $\sigma$ here depends on the (current and) past values of $S$. To achieve a complete description of the risky asset's dynamics, we only need to specify the initial values of the risky asset prior to time 0

$$
S(x)=\varphi(x), \quad x \in[-R, 0]
$$

and to assume that the system described in (2)-(3) has a unique solution (this reduces to impose that $\sigma$ is good enough ${ }^{9}$ ).

In the following, we will analyse the classical problem of pricing and hedging a contingent claim of European type with maturity $T$, whose payoff we call $H_{T}$ and assume to be a $\mathcal{F}_{T}$-measurable random variable. 


\section{Completeness of the market}

We immediately see that the fact to introduce a dynamics which depends on the past does not influence the completeness of the market, as long as we are supposing that $\left(\mathcal{F}_{t}\right)_{t}$ is a Brownian filtration. To see this, we construct, as usual, a market portfolio $V$ defined by

$$
V(t):=\Delta_{0}(t)+\Delta(t) S(t)
$$

where $\Delta_{0}$ represents the amount of money invested in the riskless asset, and $\Delta$ represents the number of shares of the risky asset held in the portfolio. We say that $V$ is self-financing if

$$
d V(t)=\Delta(t) d S(t)
$$

In this case we succeed to construct the dynamic of $V$ with the single knowledge of $V(0)$ and $\Delta$, and therefore the process $\Delta_{0}$ can be obtained consequently.

Now we turn our attention to the contingent claim $H_{T}$. We say that $H_{T}$ can be perfectly hedged if there exists a constant $V_{0} \in \mathbb{R}$ (the price) and a process $\Delta$ adapted to $\left(\mathcal{F}_{t}\right)_{t}$ (the hedging strategy) such that $V$ is self-financing and $V(T)=H_{T}$.

Proposition 3.1. (Completeness of the market) For every $H_{T}$ with finite variance, there exists a unique $V_{0}:=\mathbb{E}\left[H_{T}\right]$ and $\Delta$ such that $V(T)=$ $H_{T}$ a.s.

Proof. We define the martingale $H(t):=\mathbb{E}\left[H_{T} \mid \mathcal{F}_{t}\right]$. Then by the representation theorem of Brownian martingales, there exists a process $\xi$ adapted to $\left(\mathcal{F}_{t}\right)_{t}$ such that

$$
H(t)=\mathbb{E}\left[H_{T}\right]+\int_{0}^{t} \xi(u) d W(u)
$$

Then in order to have $V(T)=H_{T}$ it is enough to impose $V(0):=\mathbb{E}\left[H_{T}\right]$ and $\Delta(t) d S(t)=\xi(t) d W(t)$, that is verified if and only if

$$
\Delta(t):=\frac{\xi(t)}{S(t) \sigma\left(t, S_{t}\right)}
$$

Remark 3.1. As usual in continuous time complete markets, all the claims $H_{T}$ can be perfectly hedged and it can also be proved that their price at time $t$ is given by $V(t):=H(t)=\mathbb{E}\left[H_{T} \mid \mathcal{F}_{t}\right]$, as long as $\operatorname{Var}\left[H_{T}\right]<+\infty$. This is not a problem, as it can be proved that in our model (2)-(3) $S$ has finite moments of all orders $^{9}$ : this implies that all the usual claims which 
can be found on the markets (calls, puts, digitals, barrier or Asian variants, and even more sophisticated exotic options) have finite variance.

Now a problem arises: as in the models "without memory", the martingale representation theorem is not constructive: to say that "there exists a process $\xi "$ is very different to say that we know it explicitly. Moreover, in order to calculate the price we need to know the distribution of $S$. There is then the requirement to specify $\sigma$ in a way "good" enough to make explicit calculations. In order to see some practical examples, from now on we will analyse some concrete models.

\section{Pointwise dependence from the past: the Arrojas-Hu-Mohammed-Pap model}

The simplest idea for having a dependence on the past is to impose

$$
\sigma\left(t, S_{t}\right):=\sigma\left(S_{t}(-r)\right) \quad(=\sigma(S(t-r)))
$$

where $\sigma$ reduces to a real function and $r$ is a "delay" belonging to $(0, R)$. The volatility at time $t$ is therefore a deterministic function of the price of the asset at the time $t-r$ (this model is treated in Ref. 6).

This model has an explicit solution for $S$. In fact, the pointwise dependence from the past implies that in every interval $[k r,(k+1) r]$ the process is lognormal conditionally by $\mathcal{F}_{k r}$. Thus, while in principle the price of a contingent claim can be computed using the tower property of conditional expectations, it turns out that in order to calculate the price of a derivative asset in an efficient way we need to know the distribution of $S$. In particular, the marginal law of $S(t)$ is lognormal for $t \in[0, r]$, but, as long as $t>r$, it becomes more difficult to be explicitly specified (in particular, it does not belong to any known family of distributions).

Let us now turn our attention to the particular case of pricing and hedging a simple European call option. By Remark 3.1, the price is given by $V(t)=\mathbb{E}\left[(S(T)-K)^{+} \mid \mathcal{F}_{t}\right]$. Then:

- if $t \in[T-r, T]$, we obtain

$$
V(t)=S(t) N\left(d_{1}(t)\right)-K N\left(d_{2}(t)\right),
$$

where

$$
d_{1,2}(t)=\frac{1}{\bar{\sigma}_{t, T}} \log \frac{S(t)}{K} \pm \frac{1}{2} \bar{\sigma}_{t, T}, \quad \bar{\sigma}_{t, T}:=\sqrt{\int_{t}^{T} \sigma^{2}(S(u-r))^{2} d u}
$$


We have therefore a Black-Scholes type formula, with the coefficients $d_{1,2}$ depending on the "mean volatility" $\bar{\sigma}_{t, T}$, which in turns depends on all the past prices of $S$ on $[t-r, T-r]$

- if $t \in[T-2 r, T-r]$, then we can develop a recursive calculation, but it soon becomes difficult: we have in fact that $V(t)$ cannot be computed explicitly, and in order to obtain at least numerical values, the only known algorithms are Monte-Carlo methods, which are quite time-consuming.

Also for the hedging problem there are analogous problems, since it does not make sense (at least with the usual mean) to define $\Delta(t):=" \frac{\partial}{\partial S(t)} V(t)$ " as in the "not delayed" level dependent volatility models. We have:

- if $t \in[T-r, T]$, we have $\Delta(t)=N\left(d_{1}(t)\right)$;

- if $t<T-r$, problems like the ones above arise and there is not a closed formula.

\section{A model with infinite memory: the Hobson-Rogers model}

The idea of pointwise dependence presented in the previous section gives explicit results only when $T-t<r$, i.e when the time to maturity is smaller than the delay, while for a greater time to maturity we do not succeed to have explicit solutions. We now see a dependence from the past, proposed by Hobson and Rogers ${ }^{3}$, which gives better computational results.

We define for every $m \in \mathbb{N}$ the offset function of order $m$ as

$$
Z_{m}(t):=\int_{0}^{\infty} \lambda e^{-\lambda u}\left(\log \frac{S(t)}{S(t-u)}\right)^{m} d u,
$$

that can be interpreted as a measure of how much the price at time $t$ is far from all its past (the integral in fact is computed on $(0,+\infty)$ ); the exponential weight implies that the contribution of $\log \frac{S(t)}{S(t-u)}$ can be neglected when $u>>\frac{1}{\lambda}$. We then specify the volatility as

$$
\sigma\left(S_{t}(\cdot)\right):=\sigma\left(Z_{1}(t), \ldots, Z_{n}(t)\right)
$$

In this case $\sigma$ depends on the integrals (of suitable functions) of the price path of $S$ on all its past (in this case, therefore, we have $R=+\infty$ ). The advantage of this model is that, even if $S$ is not a Markov process, the process $\left(S, Z_{1}, \ldots, Z_{n}\right)$ is. In fact, by applying the Itô formula to (4), one can see that the stochastic differential of $Z$ depends only on the current values of $\left(S, Z_{1}, \ldots, Z_{n}\right)$, and not on their past. This, combined with the 
specification of the volatility in (5), implies that $\left(S, Z_{1}, \ldots, Z_{n}\right)$ is a $(n+1)$ dimensional Markov process. If the final payoff is of the form $h(S(T))$, this means that

- the price $V_{t}=\mathbb{E}\left[h(S(T)) \mid \mathcal{F}_{t}\right]$ can be computed via a PDE (as we will see later), i.e is of the form $V(t)=F\left(t, S(t), Z_{1}(t), \ldots, Z_{n}(t)\right)$, where $F$ is the solution of a suitable PDE;

- also the solution of the hedging problem is a closed formula (it is enough to use the Itô formula on $F$ and to make some computations).

In order to introduce the relevant PDE, we consider the simple case $n=1$ (we indicate for ease of notation $Z \equiv Z_{1}$ ). Then the PDE is

$$
\left\{\begin{array}{rr}
F_{t}-\lambda z F_{z}+\sigma^{2}(z)\left(\frac{1}{2} s^{2} F_{s s}+\frac{1}{2} F_{z z}+s F_{s z}-\frac{1}{2} F_{z}\right) & =0, \\
t & t<, s \in \mathbb{R}^{+}, z \in \mathbb{R} \\
F(T, s, z)=h(s) & s \in \mathbb{R}^{+}, z \in \mathbb{R}
\end{array}\right.
$$

that is a linear PDE of parabolic type, for which one can choose many numerical methods for the solution (trees, finite differences, finite or infinite elements, Monte Carlo, etc.).

\subsection{Which initial condition can we use?}

For the computation of $Z(0)$, or in general $Z(t)$, we need the path of $S$ on all its past $(-\infty, 0)$ (or $(-\infty, t)$ ). This requirement is unusual in the modelisation of financial markets, where one usually meets models that start from a certain moment in time (usually 0 ). In fact, the requirement of an infinite horizon in the past raises mathematical and "practical" (or better economical) complications:

mathematical: How can we define a stochastic calculus with time ranging on all the real line? Once that we succeed, is $Z$ well defined? In fact, remember that $Z$ is the integral of a process on $(-\infty, 0)$, so we must prove also that this integral is well defined.

economical: In the real markets, assets that "existed forever" does not exist. What we can use instead of the price path of $S$ when the asset still did not exist?

While these problems seem less worrying than stated, mainly due to the exponential weight in (4), still theoretical (and practical) solutions to these 
issues are not present in literature, at least to the authors' knowledge.

\section{A general model with finite memory}

We have seen that the Hobson-Rogers model, though having the advantage of being linked to a PDE, has some problem both from the theoretical as from the practical point of view, problems which are typical of infinite horizon models. However, we have seen that the fact to introduce integrals of the past price path can enrich the model.

Now we analyse a model where we try to keep a finite horizon and to take advantage of integrals of the price path. This model is inspired both by the Hobson-Rogers model as by a similar one in Elsanosi et al. ${ }^{7}$. In fact, notice that in the Hobson-Rogers model the offset function of order 1 can be written as

$$
Z_{1}(t)=\log S(t)-\int_{0}^{\infty} \lambda e^{-\lambda u} \log S(t-u) d u
$$

Inspired by this, we specify the volatility as

$$
\sigma\left(S_{t}\right):=\sigma(S(t), Y(t), S(t-r))
$$

where

$$
Y(t):=\int_{0}^{r} e^{-\lambda v} f(S(t-v)) d v=\int_{t-r}^{t} e^{-\lambda(t-u)} f(S(u)) d u,
$$

$f$ is a strictly monotone function and $r$ is a given delay as in the model of Sec. 4. Notice that this model is a generalisation of both the Arrojas et al. model and of the Hobson-Rogers model with only one offset function (though with a finite time horizon).

The stochastic differential of $Y$ is

$$
d Y(t)=\left(f(S(t))-e^{-\lambda r} f(S(t-r))-\lambda Y(t)\right) d t .
$$

Unlike in the Hobson-Rogers model, here the process $(S, Y)$ is not Markov, and this is more due to the finite horizon nature of $Y$ rather than to the specification of the volatility, more general than the Hobson-Rogers' one. The Markov property would be desirable, because it would be nice to reduce our model to few state variables and to reduce to PDE methods via the Itô formula. One can immediately think to use the state variables $(S(t), Y(t), S(t-r))$, but this implies a so-called anticipative stochastic calculus. In fact, by making use of the Itô formula on a deterministic function of $(S(t), Y(t), S(t-r))$, we end up with stochastic differentials of the kind $G(t, S(t), Y(t), S(t-r)) d S(t-r)$, where $G(t, S(t), Y(t), S(t-r))$ is 
not adapted to the filtration of the differential $d S(t-r)$, but "anticipates". To deal with this kind of differentials, it is necessary to use sophisticated instruments (for exampe, the Malliavin calculus), and to verify that the final results are adapted to the filtration $\left(\mathcal{F}_{t}\right)_{t}$, which can be not the case in general, to ensure that the results have the financial meaning of prices and hedging strategies which does not "see into the future".

Inspired by Ref. 7, we try to explore this shortcut: though $(S, Y)$ is not in general a Markov process, we make the strong assumption that for every final payoff of the form $h(S(T), Y(T))$, there exists a deterministic function $F$ such that:

$$
V(t)=F(t, S(t), Y(t))=\mathbb{E}\left[h(S(T), Y(T)) \mid \mathcal{F}_{t}\right]
$$

If this assumption is true, then the self-financing portfolio depends in a deterministic way only on the current values of $S$ and $Y$.

Unfortunately, the next result states that the assumption (8) is equivalent to $\sigma(s, y, z)$ not depending on $y, z$, that is to $S$ being Markov; moreover, in this case, Assumption (8) is only true for $h$ not depending on $Y$ and the function $F$ depends on $t, s$ only.

Theorem 6.1. If Assumption (8) is true, then $\frac{\partial}{\partial y} \sigma(s, y, z)=$ $\frac{\partial}{\partial z} \sigma(s, y, z)=0$.

Proof. By applying the Itô formula on $F(t, S(t), Y(t))$, we can use (2), $(6),(7)$ and the fact that the price of any claim in the market must be a $\mathbb{Q}$-martingale to obtain the PDE

$$
F_{t}+\left(f(s)-e^{-\lambda \delta} f(z)-\lambda y\right) F_{y}+\frac{1}{2} s^{2} \sigma^{2}(s, y, z) F_{s s}=0
$$

with final condition

$$
F(T, s, y)=h(s, y)
$$

Now we can follow a well known technique used in solving the PDE of affine term structure interest rates models ${ }^{10}$ : since $F$ does not depend on $z$, we can collect the terms not containing $z$, and we arrive to the conclusion that $\sigma^{2}$ must be of the form

$$
\sigma^{2}(s, y, z)=f(z) \gamma^{2}(s, y)+\bar{\sigma}^{2}(s, y)
$$

with $\gamma^{2}, \bar{\sigma}^{2}(y, z)$ being positive functions. We can collect the terms containing $z$ in (9) and arrive to

$$
F_{t}+(f(s)-\lambda y) F_{y}+\frac{1}{2} s^{2} \bar{\sigma}^{2}(s, y, z) F_{s s}=f(z)\left(e^{-\lambda \delta} F_{y}-\frac{1}{2} s^{2} \gamma^{2}(s, y) F_{s s}\right)
$$


Since this equation must hold for all the possible values of $z$, we have that the relations

$$
\begin{aligned}
F_{t}+(f(s)-\lambda y) F_{y}+\frac{1}{2} s^{2} \bar{\sigma}^{2}(s, y) F_{s s} & =0 \\
e^{-\lambda \delta} F_{y} & =\frac{1}{2} s^{2} \gamma^{2}(s, y) F_{s s}
\end{aligned}
$$

must jointly hold. Now two cases are possible: $\gamma^{2}=0$ (this means that $\sigma$ does not depend on $z$ ) and $\gamma^{2} \neq 0$. In the first case, (12) implies that $F_{y}=0$, thus (11) can be rewritten as

$$
F_{t}+\frac{1}{2} s^{2} \bar{\sigma}^{2}(s, y) F_{s s}=0
$$

Since this relation must hold for all the possible values of $y$ and $F$ does not depend on $y$, this means that $\bar{\sigma}^{2}$ does not depend on $y$, and we have the desired result.

We now claim that by assuming $\gamma^{2} \neq 0$ we arrive to an absurd. Assume $\gamma^{2} \neq 0$. Then, by eliminating $F_{s s}$ from (11)-(12) we arrive to

$$
F_{t}+\left(f(s)-\lambda y+\frac{\bar{\sigma}^{2}(s, y)}{\gamma^{2}(s, y)}\right) F_{y}=0
$$

which is a first-order linear PDE, where only the derivatives in the variables $t, y$ appear. We can thus treat the variable $s$ as a parameter, and solve in the other two. Now, it is well known from the PDE theory ${ }^{11}$ that every solution of this equation, with final condition (10), is of the form

$$
F(t, s, y)=h(s, g(t, s, y)), \quad s>0, y>0
$$

In particular, if we take $h$ to be a strictly convex function of $s$ only, we have that

$$
F(t, s, y)=h(s), \quad s>0, y>0
$$

Since $F$ does not depend on $y$, we have that $F_{y}=0$, and $F_{s s}=h^{\prime \prime}>0$. By substituting this into (12), we arrive to

$$
0=\frac{1}{2} s^{2} \gamma^{2}(s, y) h^{\prime \prime}
$$

Since $s^{2} \geq 0$ and $h^{\prime \prime}>0$, we must have that $\gamma^{2}=0$; since we started by assuming that $\gamma^{2} \neq 0$, we have an absurd.

Remark 6.1. In this failed try, we were inspired by the positive results in Ref. 7. We however have to say that in that paper the authors analyse a controlled system (which gives more degrees of freedom in reaching 
Markovianity), and also in that situation the authors succeed in reducing the system to the current values of $S$ and $Y$ only when the dynamics of $S$ is linear and with some restriction on the coefficients.

\section{Acknowledgements}

Both the authors gratefully acknowledge financial support from the CNR Strategic Project Modellizzazione matematica di fenomeni economici. The second author acknowledge financial support from the Research Training Network DYNSTOCH, under the programme Improving Human Potential financed by the The Fifth Framework Programme of the European Commission.

\section{References}

1. F. Black and M. Scholes, The pricing of options and corporate liabilities, Journal of Political Economy 81, 637-659 (1973).

2. R. C. Merton, Theory of rational option pricing, Bell Journal of Economic and Management Science 4, 141-183 (1973).

3. D. G. Hobson and L. C. G. Rogers, Complete models with stochastic volatility, Mathematical Finance 8 (1), 27-48 (1998).

4. B. Dupire, Pricing with a smile, Risk Magazine 7 (1) (1994).

5. B. Dupire, Pricing and hedging with smiles, Mathematics of derivative securities, M. A.H. Dempster (ed.) et al., Cambridge Univ. Press. Publ. Newton Inst., 103-111 (1997).

6. M. Arriojas, Y. Hu, S.-E. Mohammed and G. Pap, A delayed Black and Scholes formula, preprint (2003).

7. I. Elsanosi, B. J. Øksendal and A. Sulem, Some solvable stochastic control problems with delay, Stochastics and Stochastic Reports 71, 69-89 (2000).

8. N. El Karoui, H. Geman and J. C. Rochet, Changes of numeraire, changes of probability measures and option pricing, Journal of Applied Probability 32, 443-458 (1995).

9. X. Mao, Stochastic differential Equations And their applications, Chichester: Horwood Publications, 1997.

10. D. Duffie, Dynamic asset Pricing theory, Princeton University Press, 1996.

11. L. C. Evans, Partial differential equations, American Mathematical Society, 1998. 